\section{Escherichia coli strains with promoter libraries constructed by Red/ET recombination pave the way for transcriptional fine-tuning}

\author{
Stephan Braatsch¹, Søren Helmark², Harald Kranz¹, Brian Koebmann², and \\ Peter Ruhdal Jensen 2,3 \\ ${ }^{1}$ Gene Bridges $\mathrm{GmbH}$, Commercial Centre, Heidelberg, Germany, ${ }^{2}$ Fermenco ApS c/o \\ Technical University of Denmark, and ${ }^{3}$ Center for Systems Microbiology, Department \\ of Systems Biology, Technical University of Denmark, Lyngby, Denmark
}

BioTechniques 45:335-337 (September 2008)

doi 10.2144/000112907

System-oriented applications of genetic engineering, such as metabolic engineering, often require the serial optimization of enzymatic reaction steps, which can be achieved by transcriptional fine-tuning. However, approaches to changing gene expression are usually limited to deletion and/or strong overexpression and rarely match the desired optimal transcript levels. A solution to this all-or-nothing approach has been the use of a synthetic promoter library (SPL) that is based on randomized sequences flanking the consensus -10 and -35 promoter regions and allows for fine-tuning of bacterial gene expression. Red/ET recombination perfectly complements SPL technology, since it enables easy modification of the Escherichia coli genome and can be accomplished with linear DNA (i.e., the SPL). To demonstrate the synergistic use of Red/ET and SPL for metabolic engineering applications, we replaced the native promoter of a genomic localized phosphoglucose isomerase (pgi)-lacZ reporter construct by an SPL. Using these technologies together, we were able to rapidly identify synthetic promoter sequences that resulted in activity range of $25 \%$ to $570 \%$ of the native pgi-promoter.

The importance of Escherichia coli for biotechnology can be attributed to its fast and robust growth with minimal supplements and its defined access to DNA manipulations. Although $E$. coli is not yet entirely understood as a biological system, knowledge accumulated since the 1940s enables us to exploit it as microbial factory. Several plasmid-based expression systems have been developed for the large-scale production of heterologous protein (reviewed in Reference 1). However, metabolic engineering does not merely depend on high transcript levels of a single plasmid-borne gene; it instead requires fine control over the manipulation or de novo assembling of whole cellular pathways, such as the serial synthesis of a metabolite. These processes usually calls for chromosome engineering and precise adjustment of the enzymatic activities involved in the particular pathway.

Since the late 1990s, Red/ET recombination has represented a superior
DNA, Red/ET cloning is assisted by Red $\gamma$ (reviewed in Reference 5).

In order to manipulate E. coli gene expression, Red/ET recombineering has been used to replace entire promoter regions or $5^{\prime}$ elements [promoter, messenger RNA (mRNA)stabilizing sequence, ribosomal binding site, start codon] by initially subcloned and (error-prone) PCR-amplified sequences $(6,7)$. In bacteria, a majority of promoters possess consensus sequences known as the hexamer boxes -10 (TATAAT) and -35 (TTGACA) with which the $\sigma$-factor interacts and facilitates RNA polymerase-binding (8). Promoter tuning can be achieved by varying the nucleotides in the -10 and -35 boxes, in the spacer regions, or both. However, an oligonucleotide library where the -35 and -10 sequences are kept intact while the surrounding nucleotides are randomized results in constitutive promoters with gradual increments in strength (9-11).

To demonstrate genomic promoter fine-tuning by combining Red/ET recombineering with a synthetic promoter library (SPL) based on randomized sequences flanking the consensus -10 and -35 promoter regions, we used reporter strain GBlue, which was previously constructed by Red/ET recombineering (data not shown). In GBlue, lacZ is transcriptionally fused to the gene $p g i$, encoding a glycolytic enzyme, in the $E$. coli HS996 $\Delta$ lacX74 genetic background (Figure 1A). An SPL replacing the native $p g i$ promoter was constructed in a single step: kanamycin-resistant $\left(\mathrm{km}^{R}\right)$ cassette A002 (Gene Bridges, Heidelberg, Germany) flanked with Flp recognition target (FRT) sites was PCR-amplified using primers $\mathrm{p} 1$ and p2 (Figure 1B and Table 1). Reverse p2-type primers are degenerated oligonucleotides encoding -10 and -35 promoter consensus boxes embedded by spacers of random sequences. Previous results have shown that a 17 bp spacer sequence is a good starting point for generating a broad distribution of E. coli promoter activities with the SPL approach (9). Thus, 1775 bp PCR-amplified $\mathrm{km}^{R}$ cassettes were provided with (i) $50 \mathrm{bp}$ terminal flanks targeting them to the E. coli lysC-pgi intergenic region and (ii) promoters of 
A

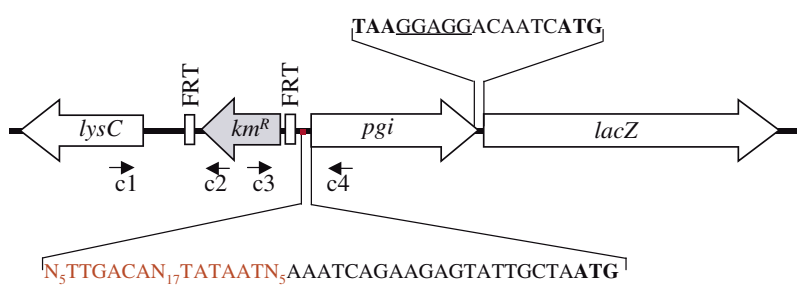

B

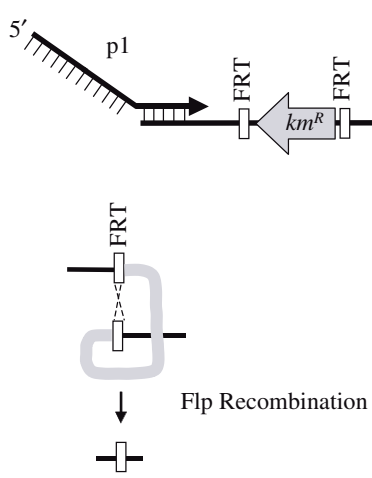

Figure 1. Construction of the pgi-lacZ fusion promoter library using Red/ET recombineering. (A) The transcriptional fused pgi-lacZ genes of GBlue were set under synthetic promoter library (SPL)-control by Red/ET recombination. (B) The synthetic promoter library (SPL) was constructed by PCR-amplifying $\mathrm{km}^{R}$ cassette A002 with primers p1 and p2 (Table 1). (C) The Flp recognition target (FRT)-flanked $\mathrm{km}^{R}$ cassette is removable by Flp-recombinase leaving a single $34 \mathrm{bp}$ FRT site at the point of DNA manipulation. Small black arrows indicate the binding sites and orientations of primers $\mathrm{c} 1-\mathrm{c} 4$, which were used to verify the structure of the recombinants and $1 / 2$, respectively. The SPL is shown in red; start (pgi, lacZ) and stop (pgi) codons are shown in bold. The synthetic lacZ ribosomal binding site is underlined.

Table 1. Oligonucleotides Used to Construct and Verify the SPL

$\begin{array}{ll}\text { Name } & \text { Sequence } \\ \text { p1 } & \text { 5'-GGGTTTACACTCAACATTACGCTAACGGCACTAAAACCATCACATTTT- } \\ & \text { TCTAATACGACTCACTATAGGG-3' } \\ \text { p2 } & \text { 5'-AGCGGTCTGCGTTGGATTGATGTTTTTCATTAGCAATACTCTTCTGATTT } \\ & \text { N }_{5} \text { ATTATAN }_{17} \text { TGTCAAN }_{5} \text { AATTAACCCTCACTAAAGG-3' } \\ \text { c1 } & \text { 5'-CAGAAAGCACAATATCAGCGC-3' }^{\prime} \\ \text { c2 } & \text { 5'-TTCTATCGCCTTCTTGACGAG-3' } \\ \text { c3 } & \text { 5'-CTGTTGTGCCCAGTCATAGC-3' } \\ \text { c4 } & \text { 5'-TTTCATCTTCTCCAGCACCG-3' }\end{array}$

Nucleotides in italics are homology arms to the targeted sequence, whereas those in standard type are the PCR primer regions; bold, synthetic promoter library (SPL); N, any nucleotide.

the $\mathrm{N}_{5}$ TTGACAN $_{17}$ TATAATN $_{5}$ type preceding the $3^{\prime}$ homology arm. The resulting $\mathrm{km}^{R}$-SPL constructs were used to replace the GBlue native $p g i$ promoter by Red/ET recombination. Strain GBlue was therefore made Red/ET-proficient by introducing pSC101-based plasmid pRed/ET (Gene Bridges). Subsequently, $200 \mathrm{ng}$ SPL cassettes were electroporated into competent GBlue (pRed/ET) cells, and $\lambda$-Red recombination was performed as described by Gene Bridges (www. genebridges.com).

About 500 colonies shaded from white to dark blue were obtained on LB plates supplemented with 15 $\mu \mathrm{g} / \mathrm{mL}$ kanamycin and $40 \mu \mathrm{g} / \mathrm{mL}$ 5-bromo-4-chloro-3-indolyl- $\beta$-Dgalactopyranoside (X-gal) implying differential pgi-lacZ expression (Figure $2 \mathrm{~A}$ inset). The $\beta$-galactosidase ( $\beta$-gal) activities of 20 selected clones were determined as described previously (12). This assay revealed a $25 \%-570 \%$ range of promoter strengths with respect to the native $p g i$ promoter of strain GBlue (Figure 2A). Correct recombinant formation was PCR-verified with primer pairs $\mathrm{c} 1 / \mathrm{c} 2$ and $\mathrm{c} 3 / \mathrm{c} 4$ (Figure 1A), which showed exclusively PCR products of the expected size of $764 \mathrm{bp}$ ( $5^{\prime}$ flank) and $1183 \mathrm{bp}$ ( $3^{\prime}$ flank). Eight representative PCR-amplified $3^{\prime}$ flanks were sequenced, revealing seven unique promoter sequences (Figure 2B) correctly positioned $5^{\prime}$ of $p g i$. The $\mathrm{km}^{R}$ cassette encoded by the complementary DNA strand $5^{\prime}$ of the synthetic $p g i$ promoters did not affect $\beta$-gal activity, as demonstrated by unchanged activity levels (data not shown) when the cassette was eliminated from the genome by Flp-mediated marker removal (Figure 1C).

The enhanced promoters identified in this study, which had activities greater than $51 \mathrm{U}$, all showed unchanged consensus boxes and normal 17 bp spacer length. In contrast, the weaker promoters with activities below $51 \mathrm{U}$ had $(i)$ a change in the -35 consensus sequence and a reduced spacer length, (ii) a gap within the -35 box, or (iii) a gap within the spacer region likely caused by integration of defective p2-type primers. The biological implication(s) of this finding remains to be solved.

With minimal effort we have been able to enhance or decrease the expression of the pgi gene transcriptionally fused to a lac $Z$ reporter along the range of a 4- to 5-fold expression change. Earlier work with $g f p$ fusions expressed from this type of constitutive promoter has shown a homogeneous distribution of fluorescence signals (13). However, to confirm that the bulk population averaged LacZ activities reflect homogenous gene expression, promoter strength needs to be determined at the single-cell level (e.g., $g f p$-based flow cytometry) as suggested by Alper et al. (6).

The combined use of Red/ET recombination and SPL enables for a one-step generation and transfer of a promoter library into the target $E$. coli strain, 
A

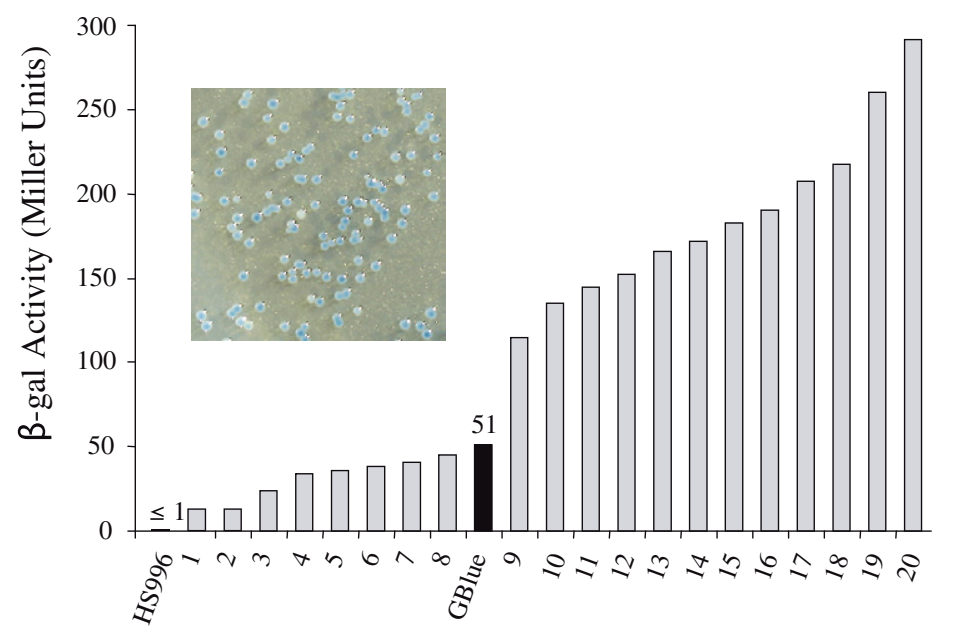

B

\begin{tabular}{|c|c|c|}
\hline Clone \# & -10 Box & $\beta$-gal Activity \\
\hline 1 & $5^{\prime}-$ TCCCTTTGATATTGCATC---CCGCGTATATAATATGTC - $3^{\prime}$ & 13 \\
\hline 3 & 5'-GCCCATTG-CATCAAGATCTGTTGAAACTATAATTCCTT - $3^{\prime}$ & 24 \\
\hline 4 & 5'-CTGCATTGA-ATAGACTTCAACTCGCGATATAATTCAAG -3' & 34 \\
\hline 7 & $5^{\prime}$ - ACACTTTGACACACCСТT-TACCCСССТTATAATTAACG - -3' & 41 \\
\hline 10 & $5^{\prime}$ - АCCTATTGACAATTAAAGGCTAAAATGCTATAATTCCAC - $3^{\prime}$ & 135 \\
\hline 15 & 5'-GGCCGTTGACAGCCCAAGCAAATACTCATATAATCATAG -3' & 183 \\
\hline 20 & $5^{\prime}$-AATACTTGACATATCACTGTGATTCACATATAATATGCG - 3' & 292 \\
\hline GBlue & $5^{\prime}-$ TGTGACTGGCGCTACAATCTTCCAAAGTCACAATTCTCA - -3' & 51 \\
\hline
\end{tabular}

Figure 2. Analysis of the activity of pgi-lacZ under control of a synthetic promoter library. (A) Promoter activity of a subset of 20 synthetic promoter library (SPL) clones, GBlue, and wild-type strain HS996, assayed via $\beta$-galactosidase ( $\beta$-gal) activity. Inset, transformants appear white to dark blue in the presence of $40 \mu \mathrm{g} / \mathrm{mL}$ 5-bromo-4-chloro-3-indolyl- $\beta$-D-galactopyranoside (X-gal) due to differential lac $Z$ expression. (B) Sequences upstream of pgi start codon of GBlue and seven representative SPL clones from subset shown in panel A. Matches to the -10 and -35 consensus boxes within the SPL sequence $\mathrm{N}_{5}$ TTGACAN $_{17}$ TATAATN $_{5}$ are highlighted in gray; gaps are indicated by dash.

thus saving both time and effort. The method allows for several markerless modifications and is applicable for optimizing gene expression in cell factories to remove bottlenecks or to reduce activities of metabolic pathways producing unwanted by-products.

\section{ACKNOWLEDGMENTS}

We are grateful to Paul R. Jaschke and Tim Zeppenfeld for helpful discussions. The authors thank Hanna Bausbacher for excellent technical assistance.

\section{COMPETING INTERESTS STATEMENT}

S.B. and H.K. are employees of Gene Bridges GmbH. S.H. is employed
5. Sawitzke, J.A., L.C. Thomason, N. Costantino, M. Bubunenko, S. Datta, and D.L. Court. 2007. Recombineering: in vivo genetic engineering in E. coli, S. enteria and beyond. Methods Enzymol. 421:171-199.

6. Alper, H., C. Fischer, E. Nevoigt, and G. Stephanopoulos. 2005. Tuning genetic control through promoter engineering. Proc. Natl. Acad. Sci. USA 102:12678-12683.

7. Meynial-Salles, I., M.A. Cervin, and P. Soucaille. 2005. New tool for metabolic pathway engineering in Escherichia coli: one-step method to modulate expression of chromosomal genes. Appl. Environ. Microbiol. 71:2140-2144.

8. Burgess, R.R. and L. Anthony. 2001. How sigma docks to RNA polymerase and what sigma does. Curr. Opin. Microbiol. 4:126131.

9. Jensen, P.R. and K. Hammer. 1998. The sequence of spacer between the consensus sequences modulates the strength of prokaryotic promoters. Appl. Environ. Microbiol. 64:82-87.

10. Jensen, P.R. and K. Hammer. 1998. Artificial promoters for metabolic optimization. Biotechnol. Bioeng. 58:191-195.

11. Solem, C. and P.R. Jensen. 2002. Modulation of gene expression made easy. Appl. Environ. Microbiol. 68:2397-2403.

12. Miller, J.H. 1972. Experiments in Molecular Genetics. CSH Laboratory Press, Cold Spring Harbor, NY.

13. Hansen, M.C., R.J. Palmer, Jr., C. Udsen, D.C. White, and S. Molin. 2001. Assessment of GFP fluorescence in cells of Streptococcus gordonii under conditions of low $\mathrm{pH}$ and low oxygen concentration. Microbiology 147:1383-1391.

Received 17 April 2008; accepted 5 June 2008.

Address correspondence to Stephan Braatsch, Gene Bridges GmbH, Commercial Centre, Im Neuenheimer Feld 584, 69120 Heidelberg, Germany. e-mail: stephan. braatsch@genebridges.com

To purchase reprints of this article, contact: Reprints@BioTechniques.com 